\title{
Computation of Electron Impact Cross Sections from Molecules of Astrophysical Importance
}

\author{
Bobby Antony \\ Department of Applied Physics, Indian School of Mines, Dhanbad, India \\ E-mail:bka.ism@in.com \\ Received January 7, 2011; revised March 12, 2011; accepted April 11, 2011
}

\begin{abstract}
Computations were performed for calculating cross sections of electron impact scattering from astrophysical targets $\mathrm{CaF}, \mathrm{HCN}$ and $\mathrm{H}_{2} \mathrm{~S}$ at energies lower than their ionization potential. The results show that the use of highly accurate R-matrix method which includes all the relevant physics and chemistry of the molecule in its formalism generate features significantly different from the simple static exchange calculation. The data obtained shows interesting structures in the eigen-phase sums which may be attributed to the dissociation channel of the molecule.
\end{abstract}

Keywords: Electron Scattering, Elasitic Cross Section, R-Matrix Method, Resonance, Dissociative Electron Attachment, Quantemol

\section{Introduction}

Considerable theoretical and experimental knowledge has now been gained on various electron scattering processes occurring with atmospheric and astrophysical molecules in gas phase. This includes the ubiquitous water molecule, for which extensive total cross section (TCS) data have been compiled recently by Itikawa and Mason [1]. The molecules in gaseous forms are known to exist in various astrophysical environments like comets, some of the planetary satellites, molecular clouds, interstellar media etc. In case of comets, electron impact on molecules is of great important due to its energetic, dynamic, and chemical consequences [2]. When the incident electrons interact with the molecules in an astrophysical environment, each of these dissociative channels can give rise to a different chemistry which is initiated by the direct and indirect collision processes [3].

Molecules like $\mathrm{LiH}, \mathrm{CaF}, \mathrm{HCN}$ and $\mathrm{H}_{2} \mathrm{~S}$ (and their cations) are believed to have played a crucial role in the early universe [4]. It has been proposed that spatial anisotropies in the cosmic radiation background (CRB) can be produced by Thompson scattering of photons from simple molecules and/or electrons located in protoclouds [5]. The efficiency of coupling between CRB photons and the primordial gas is dependent upon the chemical composition of the gas and therefore knowledge of the chemical composition of the early universe is necessary.
Lithium hydride is of particular interest since its high dipole moment makes its rotational and ro-vibrational transitions particularly strong.

One mechanism by which such ro-vibrational and dissociative transitions may be excited is electron impact, since at low energies these may be dominated by resonance formation. To date the only reported calculations are based entirely upon using the so-called static exchange approximation. In this paper we report new estimates of the elastic and electronically inelastic scattering cross sections at low energies using the R-matrix method. These calculations show that the static exchange approximation does not give reliable results for low-energy collisions and, in particular, our calculations explore the formation of temporary bound states of negative ions. Such resonances are absent in the previous studies.

\section{Theoretical Method}

The R-matrix method [6] splits configuration space into an inner region, which is a sphere of radius " $a$ " about the target centre of mass, and an outer region (Figure 1). In this work values for "a" were tested in the range $10-19$ $a_{0}$, with the results for $\mathrm{LiH}$ presented below being for the largest value. Indeed, contrary to nearly all other closed shell diatomic systems we have studied, we were unable to obtain stable results for $\mathrm{LiH}$ and $\mathrm{CaF}$ with $a<14 a_{0}$.

In the inner region the total wavefunction for the system 


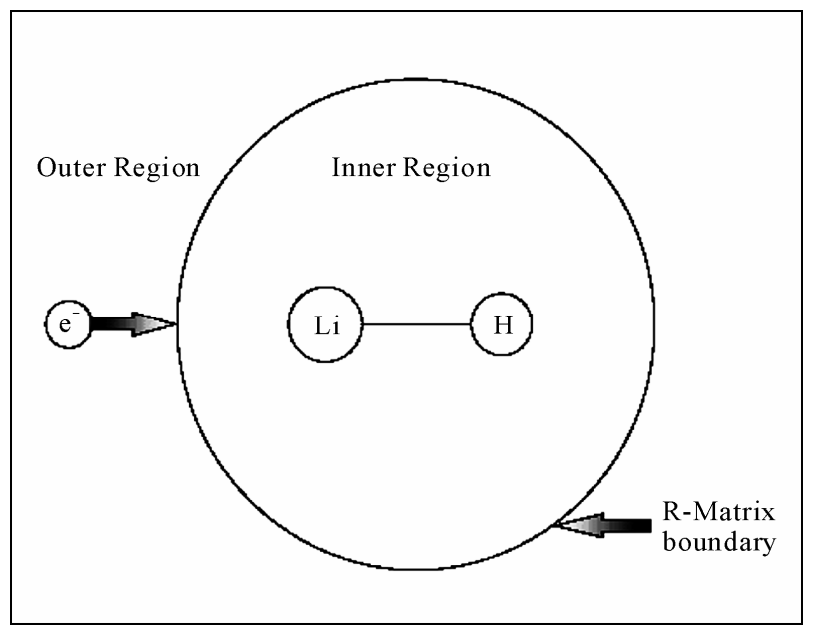

Figure 1. Division of configuration space in the fixed nuclei R-matrix theory.

is written as [7],

$$
\begin{aligned}
\psi_{k}^{N+1}= & A \sum_{I} \psi_{I}^{N}\left(x_{1}, \cdots, x_{N}\right) \sum_{j} \zeta_{j}\left(x_{N+1}\right) a_{I j k} \\
& +\sum_{m} \chi_{m}\left(x_{1}, \cdots, x_{N+1}\right) b_{m k}
\end{aligned}
$$

where $A$ is the anti-symmetrization operator, $x_{N}$ is the spatial and spin coordinate of the $n t h$ electron, $\xi_{j}$ is a continuum orbital spin-coupled with the scattering electron and $a_{i j k}$ and $b_{m k}$ are variational coefficients determined in the calculation. The first summation runs over the target states used in the close-coupled expansion. The second summation runs over configurations $\chi_{m}$, where all electrons are placed in target molecular orbitals. The number of these configurations varies considerably with the model employed. With the wavefunction given by Equation (1), a static exchange calculation has a single Hartree-Fock target state in the first sum. The second sum runs over the minimal number of configurations usually 3 or fewer, required to relax orthogonality constraints between the target molecular orbitals and the functions used to represent the configuration. Our fully close-coupled calculation uses the lowest few target states, themselves represented by a configuration interaction (CI) expansion in the first expansion and over a hundred configurations in the second. These configurations allow for both orthogonality relaxation and short-range polarization effects. It should be noted that with CI target representations, the distinction between which configurations represent which of these effects becomes blurred.

\section{Calculation}

$\mathrm{LiH}$ is a four-electron system which means that highly accurate electronic structure calculations are possible for this molecule. The target properties are obtained for $\mathrm{LiH}$ using the basis set from Salvini et al. [8] and used the Slater-type orbital (STO) basis set of Cade and Huo [9]. For other systems the basis set employed were $6-311 \mathrm{G}^{*}$ for $\mathrm{CaF}$ and DZP [6] for both $\mathrm{H}_{2} \mathrm{~S}$ and HCN. The target properties like ground state energy, dipole moment and excitation energies were found to be in good agreement with previous results.

Scattering calculations were performed using the UK molecular R-matrix program suite [6]. The outer region calculation provides the interface between the inner and outer regions. As an input this module uses data from the inner region: boundary amplitudes (MOS), eigenvalues and eigenvector of the $(N+1)$-electron Hamiltonian, target data and possibly Buttle correction. The output contains target properties, channel data and the overall symmetry of the system and data for the construction of the R-matrix and the coefficients of a multipole expansion of the long range scattering potentials.

The asymptotic solutions $j$ for each energetically open channel $i$ of the radial wave function in the outer region is given by [7],

$$
F_{i j} \sim \frac{1}{\sqrt{k_{i}}}\left[\sin \left(k_{i} r-\frac{1}{2} l_{i} \pi\right) \delta_{i j}+\cos \left(k_{i} r-\frac{1}{2} l_{i} \pi\right) K_{i j}\right]
$$

with $k_{i}^{2}=2\left(E-\varepsilon_{i}\right)$. Here $E$ is the total energy of the system and $\varepsilon$ is the eigenenergy of the ith target state. We have $F_{i j}=0$ for closed channels. The coefficients $K_{i j}$ define the real and symmetric K-matrix, containing all the scattering information. The eigenphase sum, $\delta$ used for the detection and parameterization of resonances, is found directly from the diagonalized $K$-matrix, $K_{i i}^{D}$ through,

$$
\delta=\sum_{i} \tan ^{-1}\left(K_{i i}^{D}\right)
$$

From $K$-matrix the scattering matrix $S$ is obtained by a transformation given as,

$$
S=(1+i K)(1-i K)^{-1}
$$

Then we can directly obtain $T$-matrix from the $S$-matrix as, $T=S-1$. This is used to derive physical observables such as integral and differential cross sections. The integral cross section for excitation from state $i$ to $i^{\prime}$ as given by [7] is,

$$
\sigma\left(i \rightarrow i^{\prime}\right)=\frac{\pi}{k_{i}^{2}} \sum_{S} \frac{2 S+1}{2 S_{i}+1} \sum_{\Gamma l l^{\prime}}\left|T_{i l i^{\prime} l^{\prime}}^{\Gamma S}\right|^{2}
$$

where $S i$ is the spin angular momentum of the ith target state, $S$ is the total spin angular momentum, $\Gamma$ runs over various symmetries and $l$ and $l^{\prime}$ are the orbital angular momentum quantum numbers corresponding to $i$ and $i^{\prime}$ states. In the case of electron-linear molecules scattering, $\Gamma$ is the total electronic angular momentum projected 
onto the molecular axis. Then the Born corrections are found out which adds to the total integral cross section to give the complete cross sectional data. A complete description along with different modules of the codes may be obtained from the article [10] and references therein.

\section{Results}

Our first calculation is focused on comparing results obtained with a Close-Coupling expansion with those given by the static exchange (SE) approximation (Figure 2). The eigenphase sums computed with the two models for LiH shows immense difference in its structures [11]. Present method shows structures which may be the signatures for dissociative electron attachment processes. In Figure 3 the plot of eigenphase sums for various symmetries of $\mathrm{H}_{2} \mathrm{~S}$ is depicted. Here also we can see features which are unique to R-matrix method. Since the eigenphase sums carries the signatures of scattering to any channel, it is very important to study these figures. The structures we get from these accurate calculations are the marks of specific important channel that may be present in these interactions. For example, in the case of $\mathrm{LiH}$ these structures are related to the resonance scattering.

Electron impact excitation cross sections $\left(Q_{e x c}\right)$ for $\mathrm{H}_{2} \mathrm{~S}$ were computed as a function of electron energy for the electronically excited states explicitly considered in the calculation. Results of e- $\mathrm{H}_{2} \mathrm{~S}$ elastic scattering to various excited state is presented in Figure 4. The most notable feature of these cross sections is the pronounced feature near-threshold which is present in ${ }^{3} \mathrm{~B}_{1}$ and ${ }^{3} \mathrm{~A}_{2}$ excitation cross sections. Even though these cases of excitation may not be associated with any dissociation channels, it is quite interesting. So these cross sections clearly indicate the probability of excitations to various energy levels of the molecule. Such a study can help in the analysis of electronic excitations of target due to electron impact at low energies. This is similar for other targets too, which are not shown here.

In Figure 5 we have plotted the total electron impact elastic cross section $\left(Q_{e l}\right)$ respectively for $\mathrm{CaF}, \mathrm{HCN}$ and $\mathrm{H}_{2} \mathrm{~S}$. The cross section shows very high value and hence all the structures which may be present are not visible here in this total cross section. The total elastic cross for $\mathrm{H}_{2} \mathrm{~S}$ is much lower than that of the values of $\mathrm{CaF}$ and $\mathrm{HCN}$ which seems to merge together. This feature will be interesting since they can be compared with each other and are very important molecules in astrophysical environments.

Rate coefficients for electron impact scattering of $\mathrm{CaF}$, $\mathrm{HCN}$ and $\mathrm{H}_{2} \mathrm{~S}$ molecules were presented in our Figure 6. This quantity is useful tool for further modeling of atmospheres containing such molecules. Since this quantity

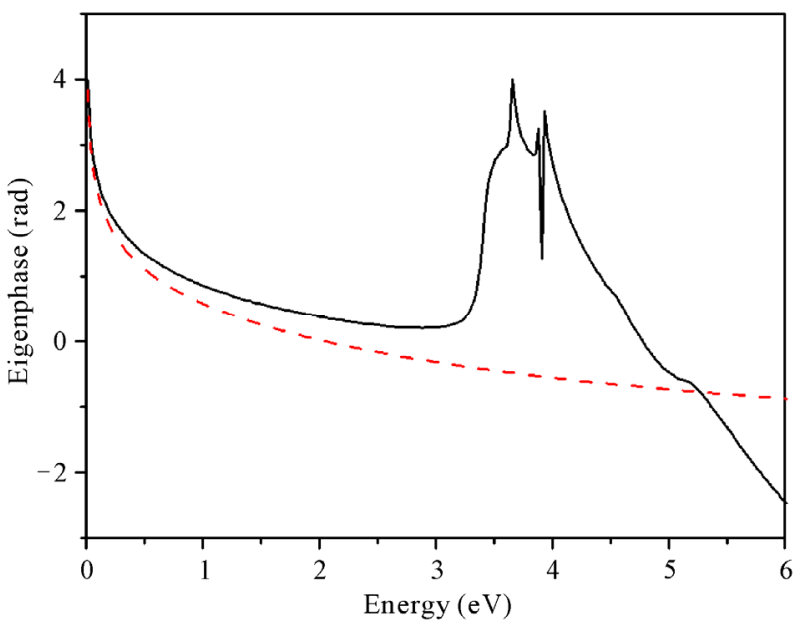

Figure 2. Eigenphase sums for e-LiH. Black-solid line present calculation; red-dashed line simple static exchange calculation.

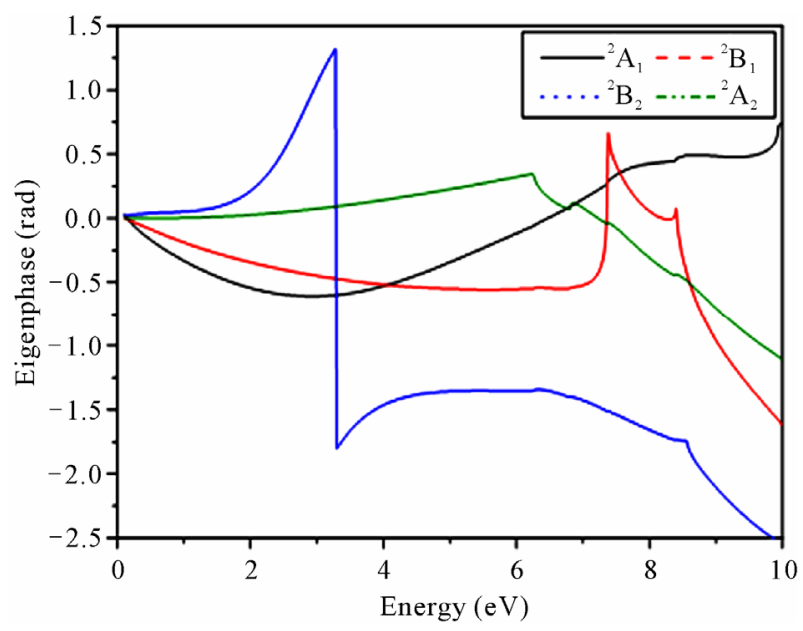

Figure 3. Eigenphase sums for various molecular symmetries of e- $\mathrm{H}_{2} \mathrm{~S}$ scattering.

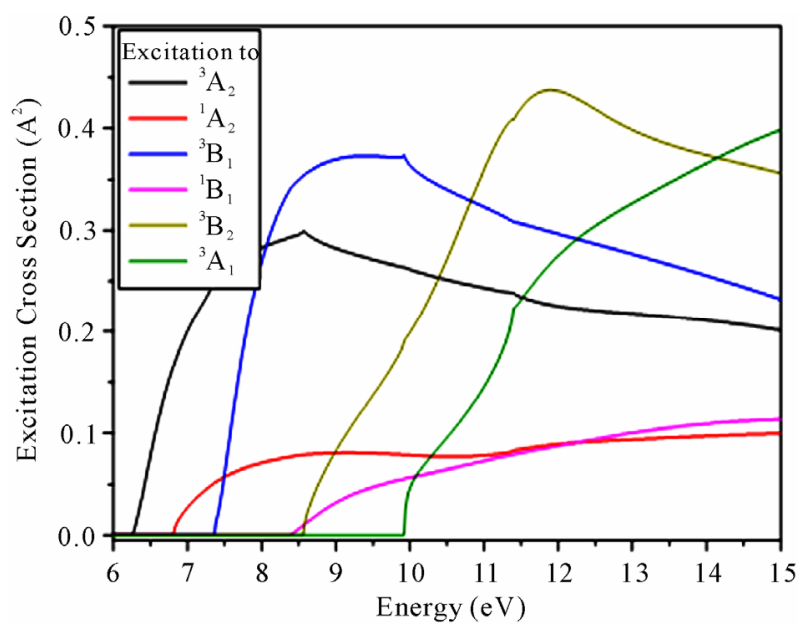

Figure 4. Low energy $Q_{e x c}$ for e- $\mathrm{H}_{2} \mathrm{~S}$ scattering to various energy levels. 


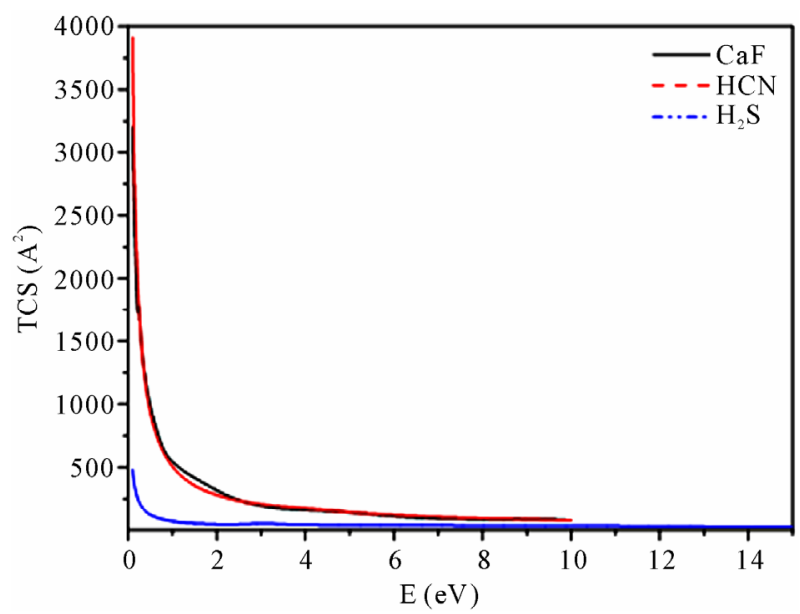

Figure 5. Low energy $Q_{e l}$ for e-CaF, $\mathrm{HCN}$ and $\mathrm{H}_{2} \mathrm{~S}$ scattering.

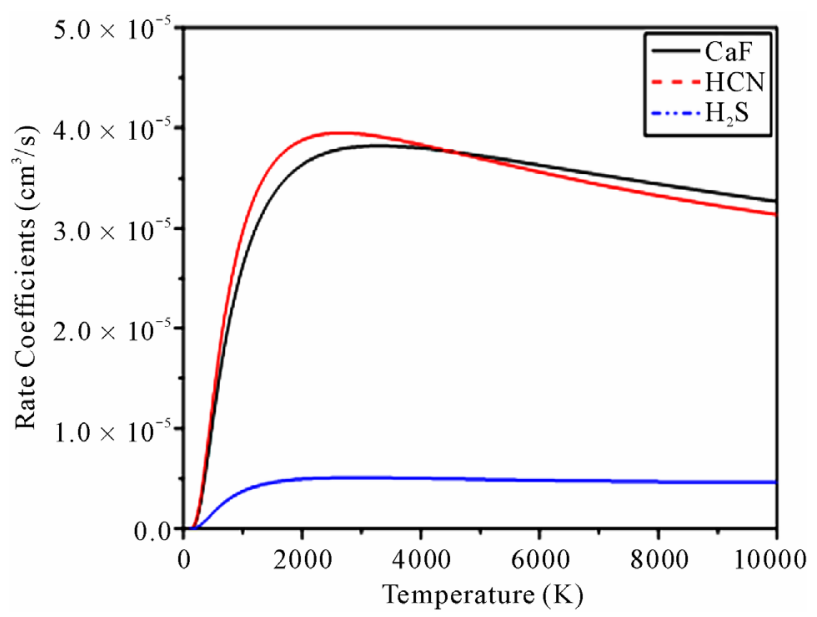

Figure 6. Rate coefficients for e-CaF, $\mathrm{HCN}$ and $\mathrm{H}_{2} \mathrm{~S}$ scattering.

is depended on the total elastic cross section, the overall comparison is very similar to the previous Figure 5. For $\mathrm{CaF}$ and $\mathrm{HCN}$ the rate coefficient is is very close to each other while for $\mathrm{H}_{2} \mathrm{~S}$ the values are much lower.

\section{Conclusions}

We have performed series of R-matrix calculations on low-energy electron collisions with important molecules like $\mathrm{CaF}, \mathrm{HCN}$ and $\mathrm{H}_{2} \mathrm{~S}$. Sophisticated models which allow for coupling with low-lying excited electronic and other target polarization effects give markedly different results from previous studies which used the static exchange approximation in which all polarization effects are neglected. Given the dominance of the electron-dipole interaction for this systems, which is correctly modeled at the static exchange level, this result confirms the observation that this model is not appropriate for treating low energy electron molecule collisions. It is such low energy, or near thermal, collisions which are important for most practical applications like astrophysics, discussed in the introduction. Our previous calculations have identified resonance features in the $\mathrm{LiH}$ system which has not previously been noted. This may lead to significant vibrational excitation and hence, it may provide a route to dissociative attachment and hence provide a low energy route for destruction of $\mathrm{LiH}$ molecules by electrons. Such a feature is missing in the present calculations confirming that dissociation may not take place at this energy range. However, such a study will have very important practical applications in the study of extra terrestrial atmospheres. Further, we hope that present investigations will trigger much enthusiasm in the experimentalists to perform better measurements at the low energy regime.

\section{Acknowledgments}

Author acknowledges the support to this research by DST, New Delhi through project No. SR/FTP/PS-27/2009.

\section{References}

[1] Y. Itikawa and N. J. Mason, "Cross Sections for Electron Collisions with Water Molecules," Journal of Physical and Chemical Reference Data, Vol. 34, No. 1, 2005, pp. 1-23. doi:10.1063/1.1799251

[2] T. E. Cravens, "X-Ray Emission from Comets," Science, Vol. 296, No. 5570, 2002, pp. 1042-1045.

[3] A. McClaine, R. W. Breault, C. Larsen, R. Konduri, J. Rolfe, F. Becker and G. Miskolczy, "Hydrogen Transmission/Storage with Metal Hydride-Organic Slurry and Advanced Chemical Hydride/Hydrogen for PEMFC Vehicles," Proceedings of 2000 US DOE Hydrogen Programme Review NREL/CP, Vol. 570, 2000, p. 28890.

[4] E. Bodo, F. A. Gianturco and R. Martinazzo, "The Gas-Phase Lithium Chemistry in the Early Universe: Elementary Processes, Interaction Forces and Quantum Dynamics," Physics Reports, Vol. 384, No. 3, 2003, pp. 85-119. doi:10.1016/S0370-1573(03)00243-6

[5] V. K. Dubrovich, "Spectral-Spatial Fluctuations of the Relic Radiation-A New Class of Objects in the Universe," Astronomical and Astrophysical Transactions, Vol. 5, No. 1-4, 1994, pp. 57-65. doi: $10.1080 / 10556799408245854$

[6] L. A. Morgan, J. Tennyson and C. J. Gillan, "The UK Molecular R-Matrix Codes," Computer Physics Communications, Vol. 114, No. 1-3, 1998, pp. 120-128. doi:10.1016/S0010-4655(98)00056-3

[7] P. G. Burke and K. A. Berrington, "Atomic and Molecular Processes: An R--matrix Approach," Institute of Physics Publishing, London, 1993.

[8] S. Salvini, P. G. Burke and C. J. Noble, "Electron Scat- 
tering by Polar Molecules Using the R-Matrix Method," Journal of Physics B: Atomic, Molecular and Optical Physics, Vol. 17, No. 12, 1984, pp. 2549-2562. doi:10.1088/0022-3700/17/12/022

[9] P. E. Cade and W. M. Huo, "Electronic Structure of Diatomic Molecules. VI.A. Hartree-Fock Wavefunctions and Energy Quantities for the Ground States of the First Row Hydrides, AH," Journal of Chemical Physics, Vol. 47, No. 2, 1967, pp. 614-649. doi:10.1063/1.1711938

[10] I. Rozum, N. J. Mason and J. Tennyson, "Electron Colli- sions with the CF Radicals Using the R-Matrix Method," Journal of Physics B: Atomic, Molecular and Optical Physics, Vol. 36, No. 12, 2003, pp. 2419-2432. doi:10.1088/0953-4075/36/12/303

[11] B. K. Antony, K. N. Joshipura, N. J. Mason and Jonathan Tennyson, "R-Matrix Calculation of Low-Energy Electron Collisions with LiH", Journal of Physics B: Atomic, Molecular and Optical Physics, Vol. 37, No. 8, 2004, pp. 1689-1698. doi:10.1088/0953-4075/37/8/010 